\title{
Estimative of the gravity-gradient data from vertical component of gravitational attraction by using the equivalent-layer and fast Fourier transform techniques
}

\author{
Larissa S. Piauilino*, Valéria C. F. Barbosa and Vanderlei C. Oliveira Jr., Observatório Nacional
}

Copyright 2019, SBGf - Sociedade Brasileira de Geofísica

This paper was prepared for presentation during the $16^{\text {th }}$ International Congress of the Brazilian Geophysical Society held in Rio de Janeiro, Brazil, 19-22 August 2019.

Contents of this paper were reviewed by the Technical Committee of the $16^{\text {th }}$ International Congress of the Brazilian Geophysical Society and do not necessarily represent any position of the SBGf, its officers or members. Electronic reproduction or storage of any part of this paper for commercial purposes without the written consent of the Brazilian Geophysical Society is prohibited.

\section{Abstract}

We present a comparison between the computing of gravity gradient tensor components from the vertical component of the gravitational attraction using the fast Fourier transform and the equivalent-layer techniques. In this comparison, we consider that the observed data consist of: (1) the residual gravity field (produced by local sources) and (2) the overlapping of regional and residual gravity fields. Applications to synthetic data show that the fast Fourier transform technique amplifies the noise to calculate the six components of the tensor. Comparing the FFT and the equivalent-layer results with the true gravity gradient components produced by local source without regional gravity field, the equivalent-layer result yields a better result because it shows a smaller residual. However, when the observed data have a regional gravity field overlapped to the residual gravity field both techniques yield unacceptable results, indicating the need of new methodologies to deal with regional field.

\section{Introduction}

One of the most difficulties in interpreting potential-field data is the presence of a regional field. The gradiometry technique has been increasingly used to facilitate the interpretation of gravity data since the great improvement of gravity gradient instruments in the 1980s. Although interpretation of six components of the gravity gradient tensor may be important in the horizontal delimitation of the sources, the interpretation of the vertical component of gravitational attraction is still easier and less costly to perform. An alternative way of estimating the components from the vertical component of the gravitational attraction is through the equivalent-layer or the fast Fourier transform techniques. However, the estimation of these components can be difficult in the presence of a regional gravity field.

The equivalent-layer is a well-established technique grounded in the potential theory. According to this theory, any potential-field data generated by a 3D source can be reproduced by a $2 D$ infinite layer of equivalent sources. Dampney (1969) showed that this layer can be represented by a discrete physical-property distribution. In the classical method, the physical-property distribution (e.g., point masses) is estimated by solving a linear system subject to fit the observed data which can be a computationally demanding process for large data sets. Therefore, some works have been made for reducing the computational cost of equivalent layer (Emilia, 1973; Hansen e Miyazaki, 1984; Silva, 1986; Leão e Silva, 1989; Cordell, 1992; Mendonça, 1994; Li, 2001; Li e Guspí e Novara, 2009; Oldenburg, 2010; Barnes, 2012; Barnes and Lumley, 2011; Oliveira Jr. et al., 2013; Barnes, 2014; Siqueira et al. 2017).

The Fourier transform technique has important properties that are useful in filter operations and linear transformations of potential field (Gunn, 1975). For processing large data sets, the computational cost is lower than the classical equivalent-layer technique. However, the data using in processing by fast Fourier transform need to be measured at a plane with constant height and in a regular grid. Besides that, the data set is extrapolated beyond the location of the observed data to reduce the edge effect.

We apply the equivalent layer and Fourier techniques to transform the vertical component of the gravitational attraction into the six tensor components. We compare the performance of these techniques in two geophysical scenarios considering the observed data are: 1) the residual gravity field (produced by anomaly sources) and 2) the overlapping of regional and residual gravity fields. Ours synthetic tests show that the equivalent layer technique yields better predicted gravity-gradient data than the Fourier transform technique when there is no regional gravity field. However, both the equivalent layer and the Fourier techniques yields worse predicted gravitygradient data than those obtained without the presence of the regional gravity field. This latter result indicates the need for modifying these techniques to deal with the presence of the regional gravity field.

\section{Methodology}

Gravity data acquired in a geophysical survey are commonly the vertical component of gravitational attractions described by the following equation (Blakely, 1986):

$$
\begin{gathered}
g(x, y, z)=c_{g} V \\
\iiint_{V} \rho\left(x^{\prime}, y^{\prime}, z^{\prime}\right) \frac{\left(z^{\prime}-z\right)}{\left[\left(x-x^{\prime}\right)^{2}+\left(y-y^{\prime}\right)^{2}+\left(z-z^{\prime}\right)^{2}\right]^{3 / 2}} d x^{\prime} d y^{\prime} d z^{\prime},
\end{gathered}
$$

where $g(x, y, z)$ is the gravity data at an arbitrary point $(x, y, z), c_{g}$ is constant factor transforming the gravity data from $\mathrm{m} / \mathrm{s}^{2}$ to $\mathrm{mGal}, \mathrm{y}$ is Newton's gravitational constant, $\rho\left(x^{\prime}, y^{\prime}, z^{\prime}\right)$ is the density of the geological body at coordinates $\left(x^{\prime}, y^{\prime}, z^{\prime}\right)$. Here, we are considering a 
ESTIMATIVE OF THE GRAVITY-GRADIENT DATA

Cartesian coordinate system $x-y-z$ with $z$ positive downward, $x$ towards the north and $y$ towards the east.

\section{The classical equivalent-layer technique}

By the equivalent-layer principle, a gravity data due to arbitrary $3 D$ source can be reproduced exactly by a continuous, infinite, 2D mass distribution. Mathematically, it is given by:

$$
\int_{-\infty}^{+\infty} \int_{-\infty}^{+\infty} m\left(x^{\prime}, y^{\prime}, z_{0}\right) \frac{g(x, y, z)=y\left(z_{0}-z\right)}{\left[\left(x-x^{\prime}\right)^{2}+\left(y-y^{\prime}\right)^{2}+\left(z-z_{0}\right)^{2}\right]^{3 / 2}} d x^{\prime} d y^{\prime}
$$

where $m\left(x^{\prime}, y^{\prime}, z_{0}\right)$ is a continuous $2 \mathrm{D}$ mass distribution at coordinates $\left(x^{\prime}, y^{\prime}, z_{0}\right)$ in which $z_{0}$ is a constant depth, with $\mathrm{z}<\mathrm{z}_{0}$.

Equation 1 is usually called as the upward continuation integral (Henderson 1960, 1970). Under practical conditions, in which an infinite discrete set of $N$ gravity observations is available and the mass distribution is a finite discrete set of $M$ equivalent source with unitary mass, equation 1 can be written in matrix notation as:

$$
\mathbf{g}=\mathbf{A m}
$$

where $\mathbf{g}$ is an $\mathrm{N}$-dimensional vector of the gravity data, $\mathbf{m}$ is an M-dimensional vector containing the physical property (e.g., mass) of each of equivalent source at depth $z_{0}$ and $\mathbf{A}$ is an $N \times M$ matrix, whose $i j$ element is the Green's function of the vertical component of the gravitational attraction observed at the coordinates $\left(\mathrm{x}_{\mathrm{i}}, \mathrm{y}_{\mathrm{i}}, \mathrm{z}_{\mathrm{i}}\right)$ caused by the $j$ th equivalent source located at the coordinates $\left(x_{j}^{\prime}, y_{j}^{\prime}, z_{0}\right)$.

In the classical equivalent-layer technique, we seek to estimate the parameter vector (mass distribution $\widehat{\mathbf{m}}$ ) on a fictitious layer of equivalent sources. The inverse problem of estimating a unique and stable parameter vector can be obtained by using zeroth-order Tikhonov regularization (Tikhonov and Arsenin, 1977):

$$
\widehat{\mathbf{m}}=\left(\mathbf{A}^{\top} \mathbf{A}+\mu \mathbf{I}\right)^{-1} \mathbf{A}^{\top} \mathbf{g}^{\mathbf{o}},
$$

where $\mu$ is the regularizing parameter, $\mathbf{I}$ is an identity matrix of order $M$ and $\mathbf{g}^{\circ} \equiv\left(g_{1}^{\circ}, \ldots, g_{N}^{\circ}\right)^{\top}$ is the $N$ dimensional observed vector of the vertical components of the gravitational attraction.

After estimating the mass distribution on the equivalent layer ( $\widehat{\mathbf{m}}$, in equation 3 ), we can compute the $N$ dimensional vector $\mathbf{g}^{\alpha \beta}$ that contains the $\mathrm{g}^{\alpha \beta}$-component of the full-tensor gradiometer by:

$$
\mathbf{g}^{\alpha \beta}=\mathbf{T} \widehat{\mathbf{m}},
$$

where $\alpha$ and $\beta \in x, y, z$ and $\mathbf{T}$ is an $N \times M$ matrix of transformation:
$T_{i j}^{\alpha \beta}=\left\{\begin{array}{ccc}\frac{3\left(\alpha_{i}-\alpha_{j}^{\prime}\right)}{r^{5}}-\frac{1}{r^{3}} & \text { if } & \alpha=\beta \\ \frac{3\left(\alpha_{i}-\alpha_{j}^{\prime}\right)\left(\beta_{i}-\beta_{j}^{\prime}\right)}{r^{5}} & \text { if } & \alpha \neq \beta\end{array}\right.$,

where $\alpha_{i}$ and $\beta_{i} \in x_{i}, y_{i}, z_{i}$ represent the $x-, y-$ and $z-$ coordinates of the ith measurement point; $\alpha_{j}^{\prime}$ and $\beta_{j}^{\prime} \in x^{\prime}, y^{\prime}, z_{0}$ represent the $x-, y$ - and $z$-coordinates of the $j$ th equivalent source and $r$ is the distance between the $t$ th measurement point and the jth equivalent source, i.e.:

$$
r=\left[\left(x_{i}-x_{j}^{\prime}\right)^{2}+\left(y_{i}-y_{j}^{\prime}\right)^{2}+\left(z_{i}-z_{0}\right)^{2}\right]^{1 / 2} \text {. }
$$

\section{The fast Fourier transform technique}

By the Fourier-convolution theorem, the $\mathrm{g}^{\alpha \beta}$-component of the full-tensor gradiometer $g^{\alpha \beta}(x, y, z)$ is given by the convolution of a function $k(x, y, z)$ and the gravity data function $g(x, y, z)$. It can be written in Fourier notation as (Blakely, 1996; Mickus and Hinojosa, 2001):

$$
\mathbf{g}^{\alpha \beta}=\mathrm{F}^{-1}\{\mathbf{K}(\mathbf{k}) \mathbf{G}(\mathbf{k})\}
$$

where $\mathrm{F}^{-1}$ represents the inverse Fourier transform, $\mathrm{G}(\mathbf{k})$ is the gravity data in Fourier domain and $\mathrm{K}(\mathbf{k})$ is an $N \times M$ matrix in Fourier domain, i.e.:

$$
\mathbf{K}(\mathbf{k})=\left[\begin{array}{ccc}
\frac{k_{\mathrm{x}}^{2}}{|\mathbf{k}|} & \frac{\mathrm{k}_{\mathrm{x}} \mathrm{k}_{\mathrm{y}}}{|\mathbf{k}|} & i \mathrm{k}_{\mathrm{x}} \\
\frac{\mathrm{k}_{\mathrm{x}} \mathrm{k}_{\mathrm{y}}}{|\mathbf{k}|} & \frac{\mathrm{k}_{\mathrm{y}}^{2}}{|\mathbf{k}|} & \mathrm{ik} \mathrm{k}_{\mathrm{y}} \\
\mathrm{ik} \mathrm{k}_{\mathrm{x}} & i \mathrm{k}_{\mathrm{y}} & |\mathbf{k}|
\end{array}\right] .
$$

where $\mathrm{k}_{\mathrm{x}}$ and $\mathrm{k}_{\mathrm{y}}$ are the wavenumbers, respectively, in the $x$ and $y$ directions and $|\mathbf{k}|=\left(k_{x}^{2}+k_{y}^{2}\right)^{1 / 2}$.

\section{Results}

To evaluate the performance of the equivalent-layer and fast Fourier transform techniques, we compared the results of true gravity gradient tensor (Figure 1) produced by a prism (horizontal projection in black lines in all Figures) with the data computed by the two techniques. Both true and predicted gravity gradient data were computed on the same vertical and horizontal coordinates of the original data shown in Figure $2 a$.

In the first test, we transformed the vertical component of the gravitational attraction $\mathbf{g}_{\mathbf{z}}$ (Figure 2a) produced by the prism (the residual gravity anomaly) to calculate the $\mathbf{g}_{\mathrm{xx}}, \mathbf{g}_{\mathrm{xy}}-\mathbf{g}_{\mathrm{xz}}, \mathbf{g}_{\mathrm{yy} y^{-}}, \mathbf{g}_{\mathrm{yz}}$ and $\mathbf{g}_{\mathrm{zz}}$-components of the gravity gradient tensor. In the second test, we simulated a firstorder regional field (Figure $2 \mathrm{~b}$ ). Then, we added this regional field to the residual field, generating a total field (Figure 2c). Thus, we calculate the tensor components with the two approaches and we evaluate the regional effect in these transformations.

To simulate experimental errors, the 2500 residual simulated data $\mathbf{g}_{z}$ were noise-corrupted with a pseudorandom Gaussian noise having zero mean and standard deviation of $0.05 \mathrm{mGal}$. This synthetic data set 
Piauilino, Barbosa ANd Oliveira JR.

was produced by one three-dimensional prism with a positive density contrast of $2670 \mathrm{~kg} / \mathrm{m}^{3}$.

To apply the equivalent-layer technique in both tests, the equivalent sources are placed at the depth of 900 meters. The predicted components of the gravity gradient tensor using the equivalent-layer technique were calculated by equations 4 and 5 , and with the estimated mass distribution ( $\widehat{\mathbf{m}}$, in equation 3 ) within the equivalent layer. The six tensor components were calculated by the fast Fourier transform technique using equations 6 and 7 .

\section{Synthetic tests 1 - residual gravity anomaly}

These tests were made to evaluate the performance of the two techniques in a simple geologic scenario where there is an isolated anomaly (without the regional gravity field). Figure 3 shows the six tensor components estimated by the equivalent layer technique. We calculated the residuals of each component defined as the difference between the observed noise-free (Figure 1) and the predicted (Figure 3 ) gravity gradient data. The six panels in Figure $4 \mathrm{a}-\mathrm{f}$ and in Figure $4 \mathrm{~g}-\mathrm{I}$ show, respectively, the residuals and the histograms of the residuals of the six components of the gravity gradient tensor. All the residuals appear normally distributed with means close to zero and with small standard deviations. Comparing with the components calculated by the fast Fourier transform technique (Figure 5), we can note an increasing in the noise. It can be confirm analyzing the residuals and the histograms of the residuals of the six components of the gravity gradient tensor in Figure 6a-f and in Figure 6g-I, respectively. All the residuals appear with standard deviation increased. This fact indicates that the equivalent-layer method can calculate the six components of the gravity gradient tensor in this scenario with a better precision than the fast Fourier transform method.

\section{Synthetic tests 2 - total gravity field}

In these tests, we evaluated the performance of the two techniques in a real geologic scenario where generally there is an overlapping of regional and residual gravity fields. Both the equivalent layer and the Fourier techniques yielded worse predicted gravity-gradient data, shown, respectively, in Figures 7 and 9, than those obtained without the presence of the regional gravity field. We can note that the edge effect increases the standard deviations of the residuals (Figures $8 \mathrm{a}-\mathrm{f}$ and $8 \mathrm{~g}-\mathrm{l}$ ) when the equivalent layer method is used. On the other hand, by calculating the six components via fast Fourier method (Figure 9) the noise is increased and the edge effect also increases the standard deviations of the residuals (Figures 10a-f and 10g-I).
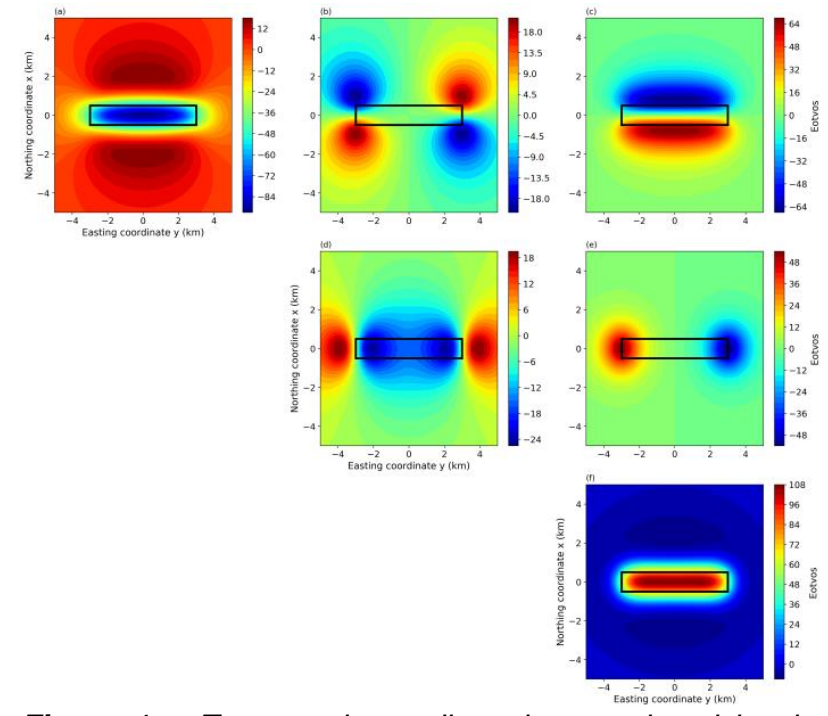

Figure 1 - True gravity-gradient data produced by the residual gravity source. The panels from left to right and top to bottom are $\mathbf{g}_{\mathrm{xx}}-, \mathbf{g}_{\mathrm{xy}}{ }^{-}, \mathbf{g}_{\mathrm{xz}}-\mathbf{g}_{\mathrm{yy}}{ }^{-}, \mathbf{g}_{\mathrm{yz}} \mathrm{z}^{-}$and $\mathbf{g}_{\mathrm{zz}}$ components of the gravity gradient tensor.
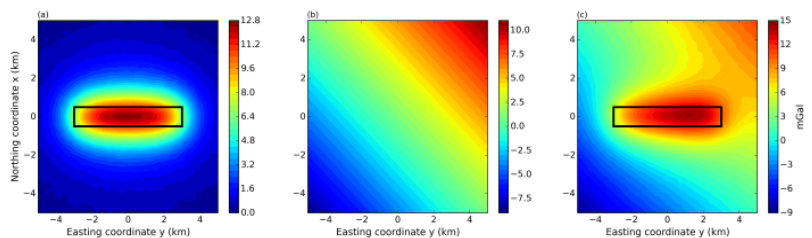

Figure 2 - (a) Noise-corrupted vertical component of the gravitational attraction produced by a prism (the residual gravity field), (b) regional gravity field simulated by a firstorder polynomial and (c) total gravity field which is the sum of the residual gravity field and the regional gravity field. The horizontal projection of the simulated prism is shown in black line.
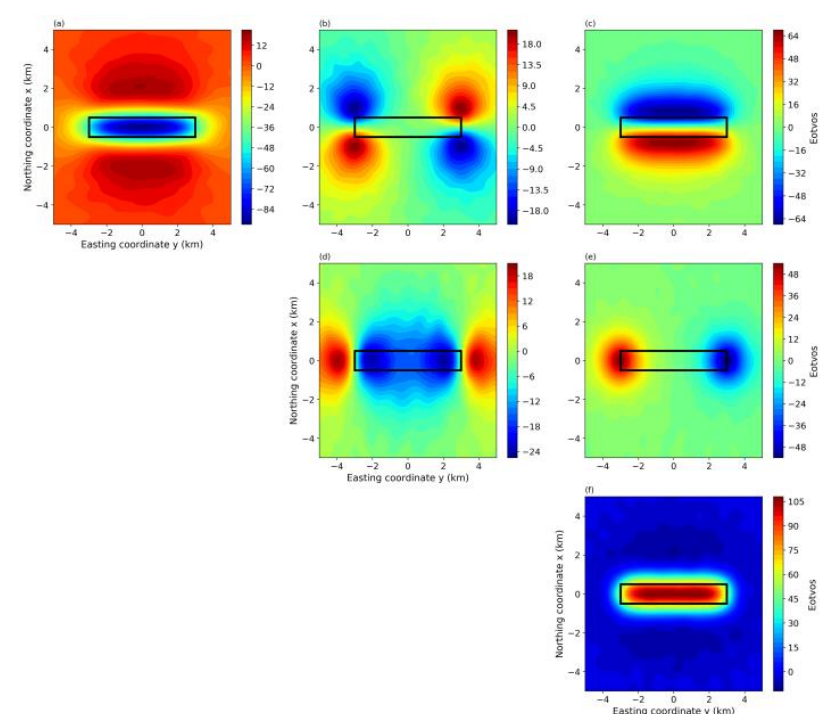

Figure 3 - Synthetic tests 1: Predicted gravity-gradient data through the equivalent-layer technique. The panels from left to right and top to bottom are $\mathbf{g}_{\mathrm{xx}}-\mathbf{g}_{\mathrm{xy}}-\mathbf{g}_{\mathrm{xz}}, \mathbf{g}_{\mathrm{yy} y^{-}}, \mathbf{g}_{\mathrm{yz}}$ and $\mathbf{g}_{\mathrm{zz}} \mathrm{z}^{-}$components of the gravity gradient tensor. The predicted components are 
ESTIMATIVE OF THE GRAVITY-GRADIENT DATA

produced by the estimated mass distribution $\widehat{\mathbf{m}}$ applying the equivalent-layer technique in residual gravity field.
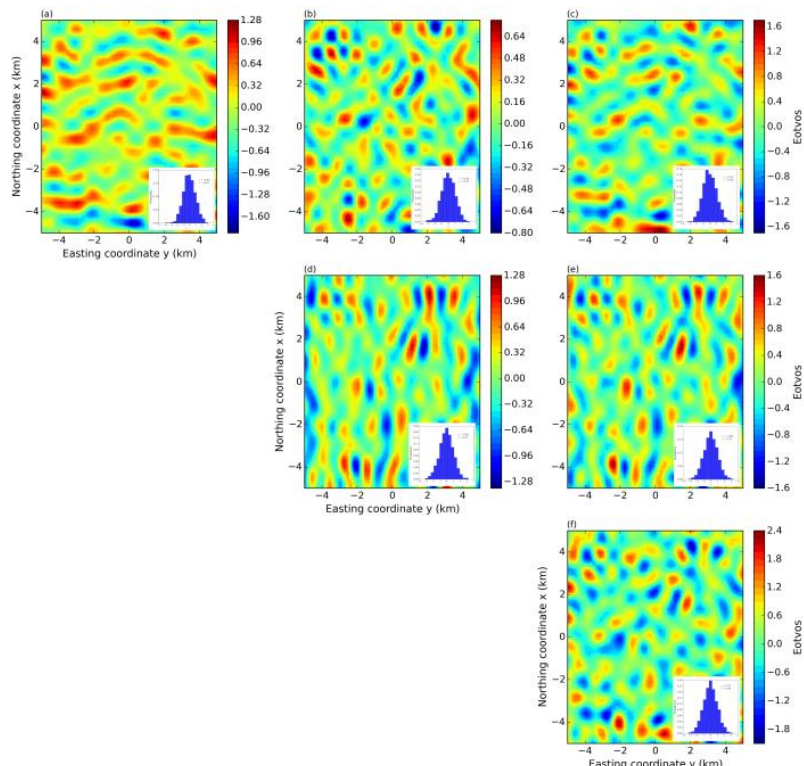

Figure 4 - Synthetic tests 1: The residuals (a-f) between the noise-free (Figure 2) and predicted (Figure 3) $\boldsymbol{g}_{x x^{-}}, \boldsymbol{g}_{x y^{-}}, \boldsymbol{g}_{x z^{-}}, \boldsymbol{g}_{y y^{-}}, \boldsymbol{g}_{y z^{-}}$and $\boldsymbol{g}_{z z^{-}}$components of the gravity gradient tensor estimated via equivalent-layer technique. Histograms of the residuals are shown as insets in (a-f) with their corresponding means $\mu$ and standard deviations $\sigma$ (in Eotvos).
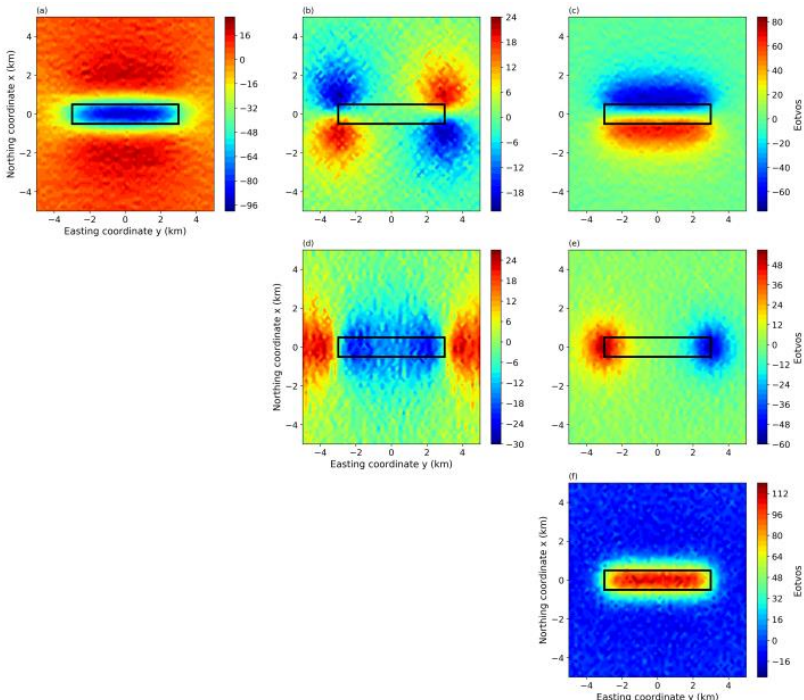

Figure 5 - Synthetic tests 1: Predicted gravity-gradient data through the fast Fourier transform technique. The panels from left to right and top to bottom are $\mathbf{g}_{\mathrm{xx}}, \mathbf{g}_{\mathrm{xy}} \mathbf{y}^{-}, \mathbf{g}_{\mathrm{xz}}, \mathbf{g}_{\mathrm{yy} y^{-}}, \mathbf{g}_{\mathrm{yz}} \mathrm{z}^{-}$and $\mathbf{g}_{\mathrm{zz}}-$ components of the gravity gradient tensor. The predicted components are produced by fast Fourier transform technique using equations 6 and 7.
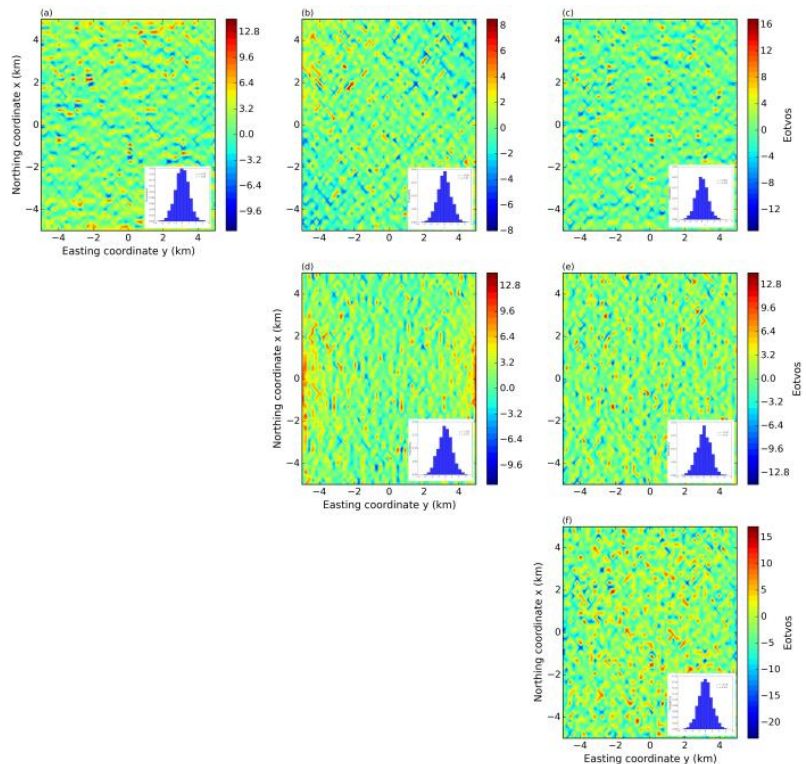

Figure 6-Synthetic tests 1: The residuals (a-f) between the noise-free (Figure 2) and predicted (Figure 5)

$\boldsymbol{g}_{x x^{-}}, \boldsymbol{g}_{x y^{-}}, \boldsymbol{g}_{x z^{-}}, \boldsymbol{g}_{y y^{-}}, \boldsymbol{g}_{y z^{-}}$and $\boldsymbol{g}_{z z^{-}}$components of the gravity gradient tensor estimated via fast Fourier transform technique. Histograms of the residuals are shown as insets in (a-f) with their corresponding means $\mu$ and standard deviations $\sigma$ (in Eotvos).
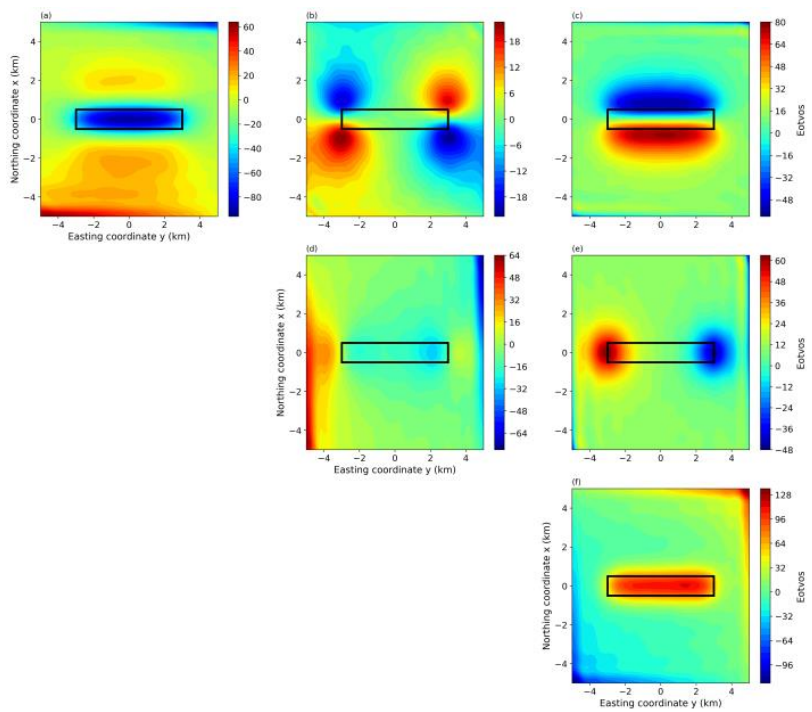

Figure 7 - Synthetic tests 2: Predicted gravity-gradient data through the equivalent layer technique. The panels from left to right and top to bottom are $\mathbf{g}_{\mathrm{xx}}-\mathbf{g}_{\mathrm{xy}}-\mathbf{g}_{\mathrm{xz}}-\mathbf{g}_{\mathrm{yy}}{ }^{-}, \mathbf{g}_{\mathrm{yz}} \mathrm{z}^{-}$and $\mathbf{g}_{\mathrm{zz}} \mathrm{z}^{-}$components of the gravity gradient tensor. The predicted components are produced by the estimated mass distribution $\widehat{\mathbf{m}}$ applying the equivalent-layer technique in total gravity field (the overlapping of regional and residual gravity fields). 

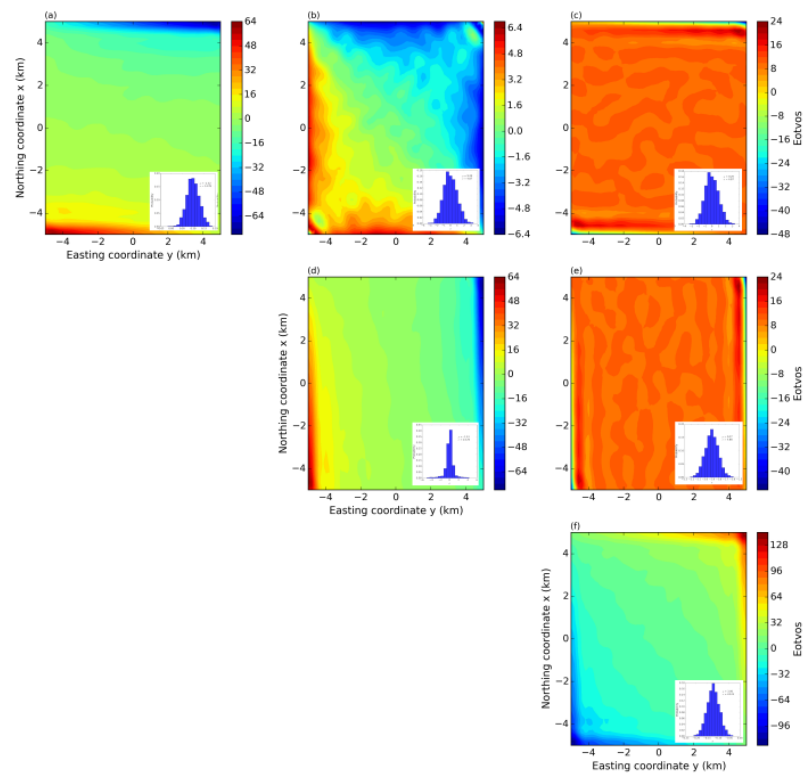

Figure 8 - Synthetic tests 2: The residuals (a-f) between the noise-free (Figure 2) and predicted (Figure 7) $\boldsymbol{g}_{x x^{-}}, \boldsymbol{g}_{x y^{-}}, \boldsymbol{g}_{x z^{-}}, \quad \boldsymbol{g}_{y y^{-}}, \boldsymbol{g}_{y^{-}}{ }^{-}$and $\boldsymbol{g}_{z z^{-}}$components of the gravity gradient tensor estimated via equivalent-layer technique. Histograms of the residuals are shown as insets in (a-f) with their corresponding means $\mu$ and standard deviations $\sigma$ (in Eotvos).
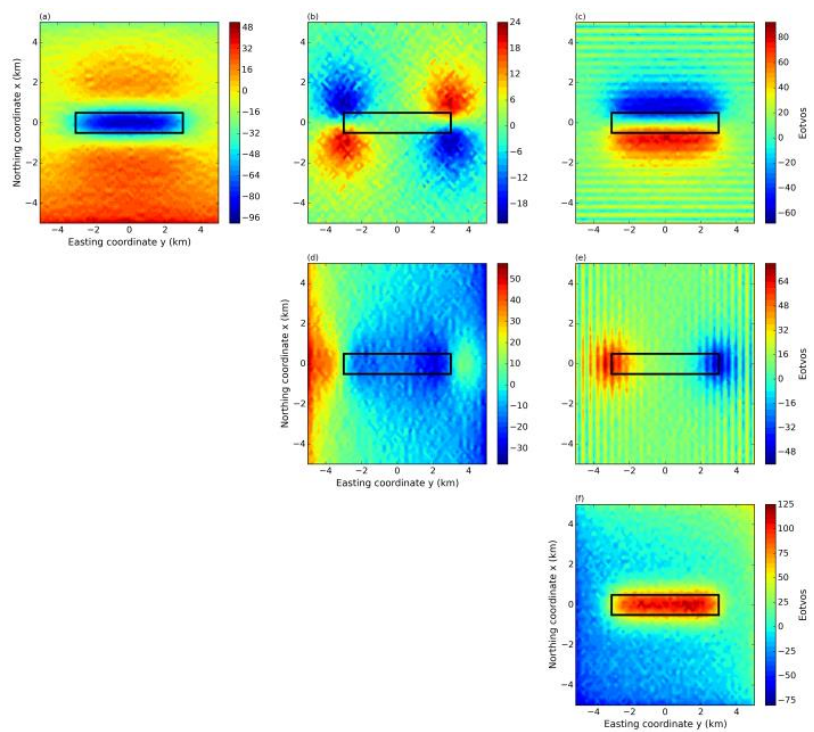

Figure 9 - Synthetic tests 2: Predicted gravity-gradient data through the fast Fourier transform technique. The panels from left to right and top to bottom are $\mathbf{g}_{x x^{-}}, \mathbf{g}_{x y^{-}}, \mathbf{g}_{x z^{-}}, \mathbf{g}_{\mathrm{yy}}-, \mathbf{g}_{\mathrm{yz}}{ }^{-}$and $\mathbf{g}_{\mathrm{zz}}-$ components of the gravity gradient tensor. The predicted components are produced by the estimated mass distribution $\hat{\mathbf{m}}$ applying the fast Fourier transform technique in total gravity field (the overlapping of regional and residual gravity fields).
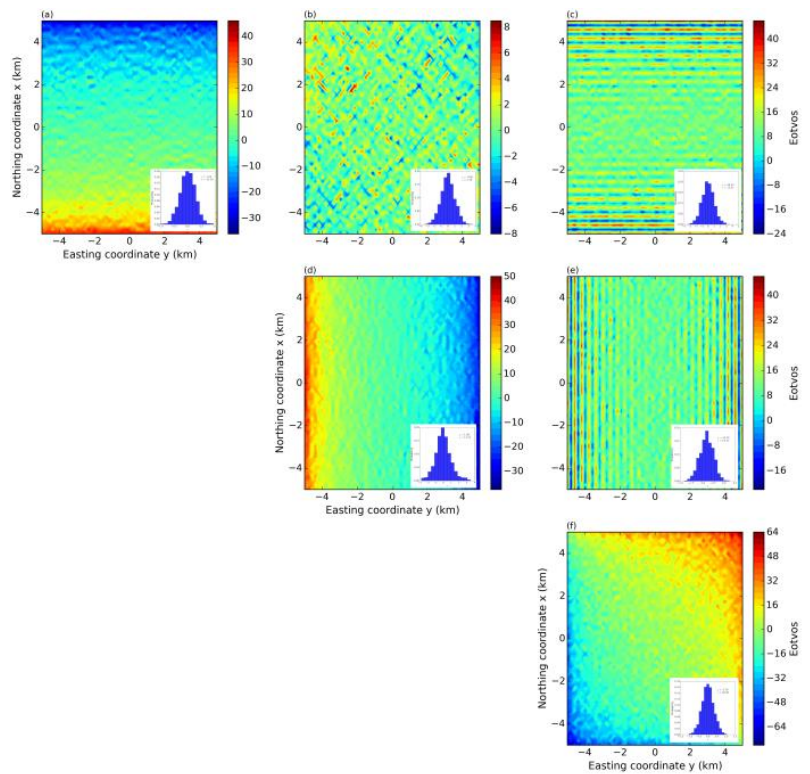

Figure 10 - Synthetic tests 2: The residuals (a-f) between the noise-free (Figure 2) and predicted (Figure 9)

$\boldsymbol{g}_{x x^{-}}, \boldsymbol{g}_{x y}, \boldsymbol{g}_{x z^{-}}, \boldsymbol{g}_{y y^{-}}, \quad \boldsymbol{g}_{y z^{-}}$and $\boldsymbol{g}_{z z^{-}}$components of the gravity gradient tensor estimated via fast Fourier transform technique. Histograms of the residuals are shown as insets in (a-f) with their corresponding means $\mu$ and standard deviations $\sigma$ (in Eotvos).

\section{Conclusions}

We have proposed the comparison between FFT and equivalent-layer method for calculating gravity-gradient data from preexisting vertical component of the gravitational attraction. In the case of residual gravity field (produced by local sources), the estimation results of the six components from an isolated gravity anomaly showed that the equivalent layer technique fits the data better than FFT technique. In data set where the regional gravity field overlaps the residual gravity field, both techniques have failed. In this case, the results obtained by the equivalent layer presented edge effects while the results of the FFT showed to be noisier and with trend lines. If a technique was used to separate regional-residual field, the equivalent-layer technique would be most appropriate for estimating the tensor components.

\section{Acknowledgments}

We would like to thank the open-source Python toolkit for geophysical modelling and inversion (Uieda et al. 2013) called Fatiando a Terra (Portuguese for Slicing the Earth) and the Coordenação de Aperfeiçoamento de Pessoal de Nível Superior (CAPES) for the financial support given to Larissa S. Piauilino through a scholarship. V.C.F. Barbosa was supported by fellowships from: CNPQ (grant 307135/2014-4) and FAPERJ (grant E-26/203.091/2016). V.C. Oliveira Junior was supported by fellowships from: CNPQ (grant 308945/2017-4) and FAPERJ (grant E26/202.729/2018). 


\section{References}

Barnes, G., 2012, Interpolating the gravity field using full tensor gradient measurements: First Break, 30, 97-101.

Barnes, G., 2014, Reconstructing the gravity gradient anomaly field from surveys with wide line spacing using equivalent source processing: an error analysis: Geophysical Prospecting, 62, 646-657.

Barnes, G., and J. Lumley, 2011, Processing gravity gradient data: GEOPHYSICS, 76, I33-147.

Blakely, R. J., 1996, Potential Theory in Gravity and Magnetic Applications. Cambridge University Press.

Cordell, L., 1992, A scattered equivalent-source method for interpolation and gridding of potential-field data in three dimensions: GEOPHYSICS, 57, 629-636.

Dampney, C. N. G., 1969, The equivalent source technique: GEOPHYSICS, 34, 39-53.

Emilia, D. A., 1973, Equivalent sources used as an analytic base for processing total magnetic field profiles: GEOPHYSICS, 38, 339-348.

Gunn, P. J., 1975, Linear transformations of gravity and magnetic fields: Geophysical Prospecting, 23, 300-312.

Guspí, F., and Novara, I., 2009. Reduction to the pole and transformations of scattered magnetic data using newtonian equivalent sources, Geophysics, 74, L67-L73.

Hansen, R. O., and Y. Miyazaki, 1984, Continuation of potential fields between arbitrary surfaces: GEOPHYSICS, 49, 787-795.

Henderson, R.G., 1960. A comprehensive system of automatic computation in magnetic and gravity interpretation, Geophysics, 25, 569-585.

Henderson, R.G., 1970. On the validity of the use of the upward continuation integral for total magnetic intensity data, Geophysics, 35, 916-919.

Leão, J. W. D., and Silva, J. B. C., 1989. Discrete linear transformations of potential field data, Geophysics, 54, 497-507.

$\mathrm{Li}, \mathrm{Y} ., 2$ 2001. Processing gravity gradiometer data using an equivalent source technique, 71st Annual International Meeting, SEG, Expanded Abstracts, 1466-1469.

Li, Y., and Oldenburg, D. W., 2010. Rapid construction of equivalent sources using wavelets, Geophysics, 75, L51L59.

Mendonça, C.A., and Silva, J. B. C., 1994. The equivalent data concept applied to the interpolation of potential field data, Geophysics, 59, 722-732.

Mickus, Kevin L., and Juan Homero Hinojosa. "The complete gravity gradient tensor derived from the vertical component of gravity: a Fourier transform technique." Journal of Applied Geophysics 46.3 (2001): 159-174.

Oliveira Jr., V. C., Barbosa, V. C. F., and Uieda, L., 2013. Polynomial equivalent layer, Geophysics, 78, G1-G13.
Silva, J. B. C., 1986, Reduction to the pole as an inverse problem and its application to low-latitude anomalies: GEOPHYSICS, 51, 369-382.

Siqueira, F. C. L., Oliveira Jr, V. C. and Barbosa, V.C.F., 2017. Fast iterative equivalent-layer technique for gravity data processing: A method grounded on excess mass constraint, Geophysics, 82, G57-G69.

Tikhonov, A. N., \& Arsenin, V. Y., 1977. Solutions of illposed problems, W. H. Winston \& Sons, Washington, DC.

Uieda L., Oliveira Jr, V. C. \& Barbosa V. C. F., 2013. Modeling the Earth with Fatiando a Terra In: Proceedings of the 12th Python in Science Conference, SciPy pp. 9198. 